\title{
Hepatectomy anesthetization for dilated cardiomyopathy patient: case report and mini review
}

\begin{abstract}
Dilated Cardio Myopathy (DCM) is a severe cardiomyopathy, characterized by systolic function decrease and dilation of the left ventricle. DCM may lead to congestive heart failure and arrhythmia, and result in sudden death. Here I report a hepatectomy anesthetization for a DCM patient. The outcome of this successful operation suggests that full preoperative preparation, delicate medication, proper intraoperative monitoring will help patients pass through perioperative period smoothly and safely.
\end{abstract}

Keywords: anesthetization, dilated cardiomyopathy, hepatectomy
Volume 6 Issue 3 - 2017

\author{
You Li, ${ }^{1,2}$ Zhu Yun, ${ }^{1,2}$ Ye Hailing, ${ }^{1,2}$ Xu \\ Pingbo, ${ }^{1,2}$ Miao Changhong 1,2 \\ 'Department of Anesthesia, Fudan University Shanghai Cancer \\ Center, China \\ ${ }^{2}$ Department of Oncology, Shanghai Medical College, Fudan \\ University, China
}

\begin{abstract}
Correspondence: Miao Changhong, Department of Anesthesia, Fudan University Shanghai Cancer Center, Department of Oncology, Shanghai Medical College, Fudan University, Shanghai 200032, China, Tel 02I-3477829I, Email miaochh@aliyun.com
\end{abstract}

Received:December 19,2016 | Published: March 06, 2017

\section{Introduction}

DCM is characterized by dilation of ventricles in mono or both sides, myocardial systolic function decrease. Many patients complicated with congestive heart-failure, arrhythmia that has close relationship with sudden cardiac death. Pathology of DCM is grossly dilated ventricles, thinned ventricular walls and interventricular septum, fibrous cicatricial tissue form tightly in the walls, and there sometimes come along with mural thrombi. Histology of CDM is nonspecific cardiac myocyte hypertrophy and degeneration, especially, the fib ration in different degree exist in mixed tissues.

Hepatectomy is an important therapy for hepatic carcinoma. Besides usual complications of hepatectomy, DCM patients have risks such as congestive heart failure, arrhythmia, low blood pressure, pulmonary edema, and the worst, sudden cardiac death.

\section{Case report}

This is a 37-year-old male, who had a history of dilated cardiomyopathy for one year. He didn't have regular medication after diagnosis. Therefore, his first visit hospital refused his operation request for poor cardiac function (LVEF $23 \%$ ). As the patient came to our hospital, we decided to treat dilated cardiomyopathy for operation preparation after multidisciplinary conference. We gave the patient digoxin $0.125 \mathrm{mg} \mathrm{qd}$, aldactone $10 \mathrm{mg} \mathrm{qd}$, irbesartan $0.15 \mathrm{~g} \mathrm{qd}$, Acertil $4 \mathrm{mgqd}$, candesartan $8 \mathrm{mg}$ qd, and some Chinese traditional medicine for 3 weeks, enhanced LEVF to $40 \%$. Abdominal CT scan indicated carcinoma located in the right lobe of liver, size was $4 \mathrm{~cm} * 3.1 \mathrm{~cm} * 4.3 \mathrm{~cm}$.

Physical examination indicated: heart rate $70 / \mathrm{min}$, respiratory rate $18 / \mathrm{min}$, blood pressure $110 / 70 \mathrm{mmHg}(1 \mathrm{mmHg}=0.133 \mathrm{kPa})$, temperature $36.7^{\circ} \mathrm{C}$, height $170 \mathrm{~cm}$, weight $65 \mathrm{~kg}$. No obvious pathologic murmurs were seen in the area of heart valve. No rhonchus and moist rales could be heard in both lungs. In addition, there was no pitting edema in both lower extremities.

Accessory examination: the cardiogram on admission suggested: sinus rhythm; low voltage in the leads of the left chest; ST segment changes (ST segment depression of $0.5-1 \mathrm{~mm}$ in leads II, III, aVF and V6); $\mathrm{T}$ wave changes (lowering of $\mathrm{T}$-wave and $\mathrm{T}$-wave indirection in leads II, III, aVF and V6); QS pattern or QR pattern occurred in leads Iand aVL. The electrocardiogram on admission indicated: left ventricular dilatation (left ventricular diastolic diameter of $70 \mathrm{~mm}$ ), decreased diffuse movement of left ventricular wall, left atrial enlargement (left atrial diameter of $43 \mathrm{~mm}$ ), LVEF $40 \%$. 24 hour dynamic electrocardiogram revealed: basic heart rate was in sinus rhythm, and the mean heart rate was 70 times $/ \mathrm{min}$, with the lowest heart rate and the fastest heart rate of 46 times $/ \mathrm{min}$ and $119 \mathrm{times} / \mathrm{min}$, respectively; meanwhile, no ischemic ST-segment changes were seen, T-wave alternans was observed.

After entering into the operating room, the patient administered oxygen inhalation and received monitoring of vital signs, the heart rate was 80 times/min and the non-invasive blood pressure was $100 / 65 \mathrm{mmHg}$. The patient was then administrated with puncturing from and catheterizing to the epidural space of T9-T10, indwelling double lumen catheter in right internal carotid, and open left upper limb vein at the same time ( $16 \mathrm{~g}$ indwelling needle) to monitor invasive blood pressure of left radial artery, Vijileo displayed $\mathrm{CO}$ of 7.9 and SVV of 8 . Before anesthesia induction, the patient was administrated with loading dose of milrinone $(0.375 \mathrm{mcg} / \mathrm{kg} / \mathrm{min})$. Induction drugs: midazolam of $1 \mathrm{mg}$, etomidate of $20 \mathrm{mg}$, diprivan of $1 \mathrm{mcg} /$ $\mathrm{ml}$, sulfentanyl of $20 \mathrm{mcg}$, esmeron of $50 \mathrm{mg}$, and dexamethasone of $5 \mathrm{mg}$. The intubation was successful. Maintenance of anesthesia: $2 \%$ sevoflurane, Epidural $0.25 \%$ naropin $(4 \mathrm{ml} / 45 \mathrm{~min})$, combined with intermittent administration of phenylephrine to maintain the stable circulation. Equilibrium liquid of $1000 \mathrm{ml}$ and hydroxyethyl starch of $1000 \mathrm{ml}$. CO fluctuation during operation ranged from 6.5 to 9.5 , SVV controlled between 4-15, the fluctuation of heart rate in 60-90 times/ min, and systolic blood pressure fluctuation in $100-140 \mathrm{mmHg}$. The length of operation time was 1.5 hours, the amount of bleeding was about $300 \mathrm{ml}$, and the urine volume was $100 \mathrm{ml}$.

Extubation was performed 15 minutes after operation when the patient was awake, the patient had a sense of fatigue and sleepiness, and showed transient decreases in blood pressure, following PACU observation 1 hour later, the patient returned to ICU intensive care. 
After 2days stay in ICU, the patient transferred to general ward. And the patient discharged hospital 6 days after operation.

\section{Discussion}

In patients with dilated cardiomyopathy, sudden death may occur in perioperative period, and the prevention of sudden death is mainly the control of reversible factors of ventricular arrhythmia, for example, to correct heart failure and electrolyte imbalances, to improve neurohormonal disorders and select ACEI and $\beta$-blockers, and to avoid drugs relevant side effects.

Warfare should be administrated since patients with dilated cardiomyopathy are prone to forming extracardiac mural thrombus with extracardiac blood stasis $^{2}$ all cardiac cavities significantly enlarged with decreased ejection fraction, grade IV heart function, long-term bed and previous history of thromboembolism, patients with deep venous thrombosis, atrial fibrillation and symptomatic heart failure should take anticoagulants orally. Atrial fibrillation patients without contraindication can receive oral administration of aspirin to prevent the formation of mural thrombus, meanwhile, patients with mural thrombus and thromboembolism development must receive long-term anticoagulant therapy, oral administration of warfarin, and the adjusted dose was made and maintained between 2-2.5 according to the international normalized ratio (INR).

Here the patient with dilated cardiomyopathy completed right liver resection successfully under epidural anesthesia combined with general anesthesia. Preoperative cardiac function was carefully assessed, and coronary artery stenosis was assessed by coronary CTA examination. ${ }^{3}$ Induction of slow anesthesia was made under continuous invasive arterial pressure monitoring, try to avoid drugs that have an inhibitory effect on the heart. During the maintenance period of anesthesia, it is necessary to maintain the cardiac systolic function and the proper use of milrinone ${ }^{4}$ or other inotropic drugs, regulate heart preload and the control afterload, as well as avoid hypotension, tachycardia and arrhythmia. Strictly monitor the capacity status of patients, maintain volume, electrolyte and acid-base balance. During epidural anesthesia, dilated cardiomyopathy patients should be administrated with low concentration and small dose of local anesthetics to avoid sudden hypotension. Anesthesia induction must be slow and careful, anesthetic thatinhibit myocardial contraction, such as remifentanil, should be avoided. Etomidate, fentanyl, vecuronium bromide are preferred. ${ }^{5}$ Early use of positive inotropic drugs can be applied for circulatory supports to shorten the hypotension continue time after induction. Simultaneously, avoid circulatory inhibition caused by excessive anesthesia, and thereby induce intractable hypotension.
Due to myocardial fibrosis, dilated cardiomyopathy patients may involve and affect cardiac pacemaker conduction system, which may easily lead to arrhythmia. ${ }^{6}$ The dominant manifestation of arrhythmia is ectopic cardiac rhythm and premature ventricular contraction in particular; the incidence of atrial fibrillation is about $10-30 \%$; there are also various types of conduction block, and right bundle branch block is the most common one. Patients who have arrhythmia can choose medicine according to different arrhythmia, among which amiodarone is a relatively safe and effective antiarrhythmic drug. Patients with symptomatic bradycardia will probably require cardiac pacemaker implantation. Furthermore, automatic implantable cardiac defibrillator may be considered in patients with severe left ventricular dysfunction and/or sustained ventricular tachycardia.

\section{Acknowledgments}

None.

\section{Conflicts of interest}

The authors declare there is no conflict of interests.

\section{Funding}

None.

\section{References}

1. Lowes BD, Gilbert EM, Abraham WT, et al. Myocardial gene expression in diated cardiomyopathy treated with beta-blocking agents. New Engl Jo of Med. 2002;346(18):1357-1365.

2. Tsevat J, Eckman MH, Mcnutt RA, et al. Warfarin for dilated cardiomyopathy: a bloody tough. Med Decis Making. 1989;9(3):162169.

3. M Francone. Role of cardiac megnetic resonance in the evaluation of dilated cardiomyopathy: diagnostic contribution and Prognostic significance. Isrn Radiology. 2014:365404-365404.

4. Abu-Shahwan I. Is sevoflurane and remifentanil induction of anesthesia safe in children with severe dilated cardiomyopathy?. J Cardiothorac Vasc Anesth. 2008;22(5):744-745.

5. Raj R, Kumar M, Batra M. Anaesthetic management of a case of dilated cardiomyopathy for emergency appendectomy. Anesth Essays Res. 2014;8(1):105-107.

6. Unverferth DV, Baker PB, Swift SE, et al. Extent of myocardial fibrosis and cellular hypertrophy in dilated cardiomyopathy. Am J Cardiol. 1986;57(10):816-820. 